\title{
Esperienza nell'utilizzo di un nuovo presidio per il Priming dei CVC nel trattamento di Emodialisi
}

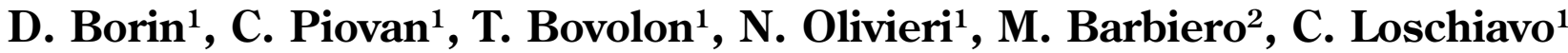 \\ ${ }^{1}$ U.O.C. di Nefrologia e Dialisi, Ospedale di Legnago \\ ${ }^{2}$ U.O.S.V.D. di Terapia Intensiva Cardiologica, Ospedale di Legnago (VR)
}

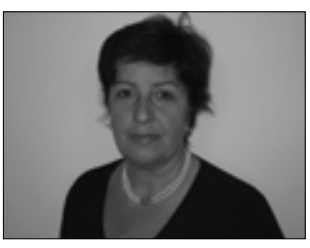

Donata Borin

\section{Background}

Per ottenere un trattamento emodialitico adeguato è necessario un accesso vascolare che fornisca un flusso ematico sufficiente a garantire l'adeguatezza della seduta dialitica e la fistola rimane l'accesso vascolare elettivo per i pazienti cronici in emodialisi (1). Il catetere venoso centrale (CVC) è utilizzato nei pazienti in attesa di allestimento di fistola arterovenosa o nei pazienti nei quali non è possibile creare un accesso chirurgico con vasi nativi o con protesi vascolare.

Il CVC è inserito in una vena centrale (giugulare interna, femorale, e un tempo nella succlavia) ed è preferibilmente a due lumi, per consentire la contestuale aspirazione e restituzione del sangue che attraversa il circuito extracorporeo.

Per l'invecchiamento della popolazione dialitica e per l'aumento delle comorbidità (diabete mellito, vasculopatie generalizzate, scompenso cardiaco ecc.), gli accessi venosi centrali sono diventati una componente integrante delle procedure di accesso vascolare per l'emodialisi e, anche se le linee guida DOQI (2) raccomandano che l'uso del catetere sia mantenuto a livelli inferiori al 10\% dei pazienti in emodialisi croni$\mathrm{ca}$, in molti centri la loro prevalenza ha di gran lunga superato questo limite; negli Stati Uniti e nel Regno Unito, rispettivamente $18 \%$ e $24 \%$, secondo lo studio DOPPS (3).

Validi motivi giustificano l'uso prolungato dei cateteri per dialisi nel periodo di attesa della maturazione della fistola arterovenosa o di un rene da donatore vivente $o$, sempre di più, come accesso vascolare permanente in pazienti che hanno esaurito tutte le possibilità di un accesso vascolare con vasi nativi, che peraltro sono ancora gli accessi emodialitici di riferimento quando possibili.

Il CVC costituisce tuttavia anche una potenziale fonte di batteri dall'esterno verso il circolo e per questo motivo è buona pratica clinica eseguire le manovre di inserimento e di gestione del presidio con tecnica sterile.

Le infezioni e la trombosi totale rimangono, infatti, le principali complicanze che possono compromettere la dialisi stessa e limitano l'emivita del catetere (4).

Per prevenire la trombosi del CVC viene impiegata l'eparina a diverse concentrazioni o diluita o in alternativa il Citrato di Sodio a diverse concentrazioni (5-8).

La nostra esperienza ci ha portato a sostituire completamente l'eparina con il citrato $(3,8 \%)$, per evitare le ben note complicanze (trombocitopenia, problematiche coagulative/emorragiche) $(9,10)$, anche per l'indiscutibile vantaggio economico.

Tuttavia allo stato attuale nessuna procedura o utilizzo di sostanze con spiccata attività anticoagulante è in grado di garantire la pervietà del catetere, e pertanto di escluderne l'occlusione, prima dell'avvio della procedura dialitica.

\section{Occlusione e malfunzionamento del CVC}

L'occlusione del CVC può essere dovuta a coaguli di sangue refluito all'interno del CVC (è l'evidenza più frequente), aggregati lipidici in corso di NPT, assai più raramente depositi di minerali, il malfunzionamento può essere dovuto a strozzamento, come nel 
pinch-off (pinzamento del CVC tra la clavicola e la prima costola) e nel kinking (inginocchiamento); in questo caso la risoluzione, se possibile, avviene sotto scopia.

Un tipo particolare di occlusione è la withdrawal occlusion, cioè l'impossibilità di aspirare sangue dal catetere, mentre la possibilità di infondere è conservata; è dovuta per lo più a una guaina di fibrina che si forma nella punta del catetere. Il recupero della funzione può essere ottenuto, talvolta, con energiche aspirazioni capaci di rimuovere la guaina che, con meccanismo a valvola, ostacola il prelievo. Se il tentativo è infruttuoso, si può ricorrere a un dispositivo usato per il brushing endoluminale a scopo diagnostico per le infezioni associate al CVC.

Lo specolo di tale presidio, spinto oltre la punta del $\mathrm{CVC}$, quando viene ritirato, trascina con sé la guaina di fibrina e risolve l'occlusione in aspirazione, si tratta, tuttavia, di un metodo costoso.

É pratica clinica comune intervenire anche mediante l'iniezione di sostanze ad attività trombolitica (urokinasi, etanolo o acido cloridrico) e tale intervento di disostruzione del catetere è da considerare pratica medica.

Nel caso in cui non si riesca ad iniettare la sostanza è preferibile rinunciare alla disostruzione e far sostituire il presidio.

\section{Preparazione e Testing del CVC allo stato attuale}

La maggioranza dei protocolli in uso per la preparazione del CVC all'inizio della dialisi prevede l'impiego di quattro siringhe di medio calibro $(20 \mathrm{~mL})$ per la disostruzione e il test delle vie del catetere prima dell'attacco.

Il numero di manovre eseguite non è costante poiché dipende dalla manualità e dall'esperienza dell'infermiere e non è stato disponibile, sino ad ora, un metodo che dia sufficienti garanzie di rimozione del coagulo e che permetta di valutare la portata del catetere stesso.

La prima operazione, condotta per ognuna delle due vie del catetere, è l'aspirazione di circa $10 \mathrm{~mL}$ di sangue mediante una siringa da 10 o da $20 \mathrm{~mL}$ allo scopo di aspirare, contemporaneamente alla Locking solution, l'eventuale coagulo.

L'avvenuta rimozione viene generalmente dimostrata visualizzando il coagulo (che potrebbe anche non essersi formato in funzione dell'assetto coagulativo del paziente) dopo aver espulso il sangue all'interno di un contenitore. Con questa manovra viene persa una quantità di sangue che si aggira tra i 10 e i $15 \mathrm{~mL}$. Nel caso in cui il coagulo non sia visibile si possono eseguire più aspirazioni con ulteriore perdita significativa di sangue del paziente. Ciò nonostante, in considerazione del comunque esiguo volume movimentato, non vi è garanzia certa che sia avvenuta l'aspirazione dell'eventuale coagulo.

In seguito si passa a una valutazione empirica della portata mediante 2-3 aspirazioni con siringa, riempita con $10 \mathrm{~mL}$ di fisiologica; quindi il sangue, misto a fisiologica, viene restituito totalmente.

Queste operazioni comportano, oltre a una perdita ematica, una serie non prevedibile di connessioni e sconnessioni con i relativi rischi infettivi per il paziente, considerando anche unicamente la non-sterilità dell'ambiente e la perdita di sterilità legata alle connessioni-sconnessioni multiple.

Il rischio infettivo è ovviamente elevato anche per l'operatore poiché nelle condizioni descritte è presente sangue potenzialmente infetto in ambiente.

Per ultimo, la procedura descritta non consente una corretta valutazione della portata dell'accesso nella configurazione di funzionamento, il che significa che alla partenza della seduta dialitica potrebbe essere necessario eseguire nuove sconnessioni per ri-testing e lavaggio e a questo punto è necessario fermare la procedura già avviata e ritardare ulteriormente l'inizio della seduta.

Da queste verifiche ed osservazioni emerge la necessità di individuare una metodica o un sistema che consenta il miglioramento delle attuali tecniche di manutenzione $\mathrm{e}$ controllo del funzionamento del CVC.

\section{“Haemocatch" Bilume: Sistema per il Priming ed il testing del CVC per Emodialisi}

Recentemente è stato introdotto sul mercato un medical device sterile, denominato Haemocatch, composto da elementi plastici attivi e passivi con l'obiettivo di eseguire tutte le operazioni in precedenza elencate in sicurezza e semplicità.

Il device (Fig. 1) è composto da:

- Connessione luer-lock con auto-espulsione allo svitamento

- Ypsilon flessibile per facile connessione al catetere

- Valvola unidirezionale di ritorno sangue filtrato

- Filtro da $50 \mu$ in nylon

- Vaschetta comprimibile e collassabile a circa -250 $\mathrm{mmHg}$ 


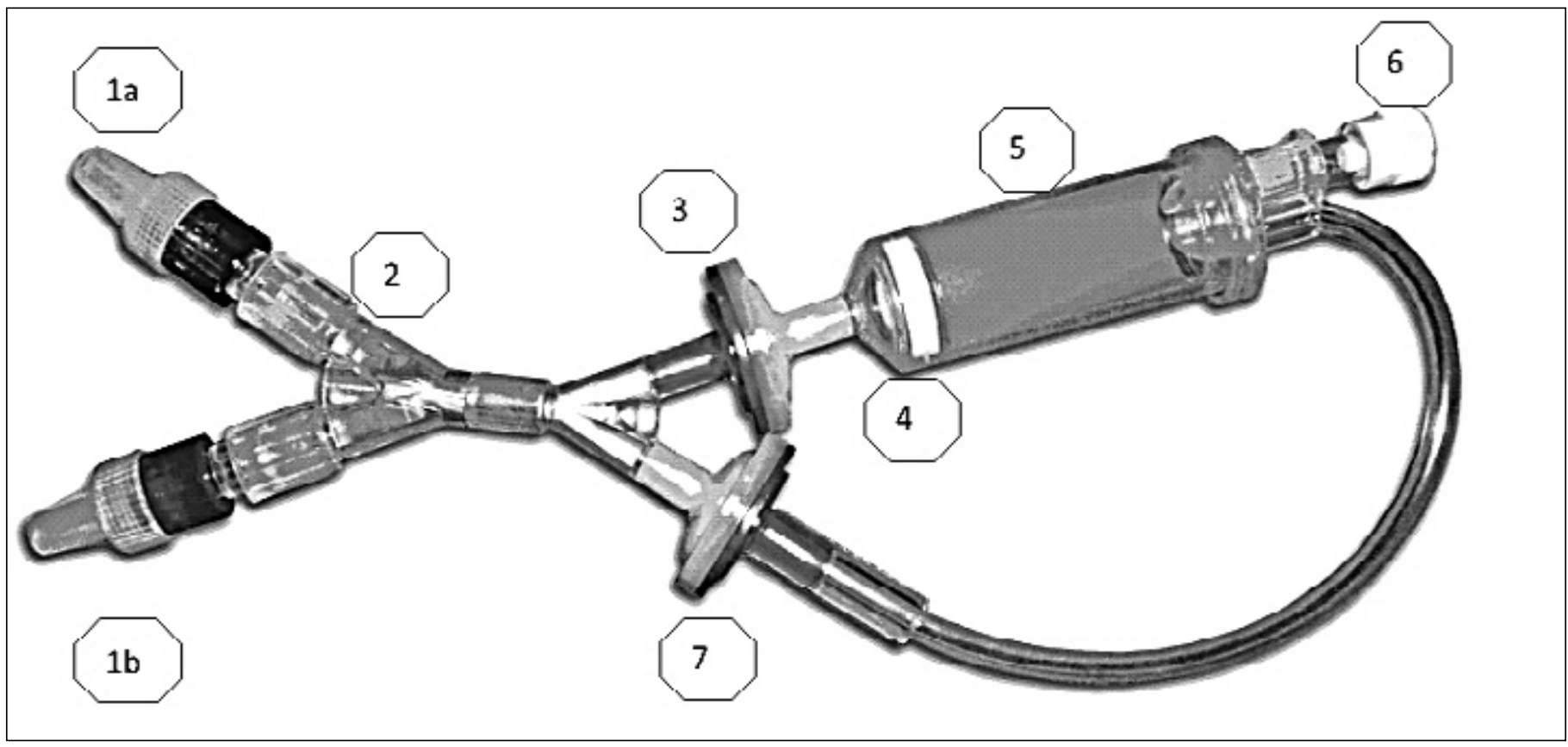

Fig. 1 - Haemocatch bilume.

- Connessione Luer per siringa da $20 \mathrm{~mL}$

- Valvola unidirezionale di aspirazione

Le operazioni che il device consente di effettuare sono fondamentalmente tre:

- Aspirazione del sangue da entrambi i lumi (in sequenza)

- Restituzione del sangue filtrato e privo di eventuali coaguli o agglomerati di fibrina

- Test di portata dell'accesso vascolare.

\section{Operatività nell'utilizzo del device}

Per quanto riguarda la soluzione di Locking (LS) il device attualmente non prevede una manovra dedicata e pertanto è l'operatore che, mediante un opportuno protocollo a seconda della soluzione utilizzata nell'ultima seduta dialitica, deve comportarsi di conseguenza. Nella maggior parte dei nostri casi la "chiusura" del catetere, dopo opportuno lavaggio, avviene con citrato di sodio isotonico a bassa concentrazione $(3,8 \%)$ iniettabile (Ph Eur.) e tale LS non viene rimossa $(6,11)$ riducendo a sole due connessioni l'intera operatività. In tutti gli altri casi è invece previsto l'utilizzo di 2 siringhe da 2,5 mL per la sola rimozione del volume della LS che equivale, nella maggior parte dei casi, a poco più di un $\mathrm{mL}$ per lume.

\section{L'utilizzo del device si fonda su due fasi: una prima fase "preparatoria" e una seconda fase "esecutiva"}

Prima fase: Una siringa luer lock da $20 \mathrm{~mL}$ riempita con fisiologica è connessa al punto di accesso (Fig. 1, punto 6) del device per il priming. Si posiziona poi il set verso l'alto e si inietta molto lentamente la fisiologica sino al riempimento dello stesso. Successivamente si completa il lavaggio del device portando a fine corsa lo stantuffo della siringa su un contenitore aperto.

Seconda fase: Si collega l'aspirazione (1 a) alla via arteriosa e il ritorno ( $1 \mathrm{~b})$ alla via venosa del catetere mantenendo il set a una angolazione di $30-45^{\circ}$.

Dopo aver aperto la clamp della via venosa del catetere, si crea una rapida depressione con la siringa fino al suo totale riempimento per poi restituire il sangue portando a fine corsa lo stantuffo.

Si testa poi la portata del lume venoso come descritto nel paragrafo "Test di portata" (vedi oltre) e si clampa la via venosa.

Si apre poi la clamp della via arteriosa del catetere e si crea una rapida depressione con la siringa fino al suo totale riempimento.

Si effettua il test di portata del lume arterioso come descritto nel paragrafo "Test di portata" e si clampa la via arteriosa.

Si procede successivamente, come di consueto, con 
il collegamento delle linee AV.

Tutte queste operazioni si svolgono nell'arco di un minuto e si riassumono in: priming, verifica valvole, aspirazione sangue, test di portata e restituzione.

\section{Test di Portata/Flowrate (Q) del catetere}

Si definisce portata il volume di fluido che attraversa la sezione di un condotto nell'unità di tempo; nel nostro caso la portata è il rapporto tra il volume che passa attraverso il lume e il tempo che lo stesso volume impiega a passare:

$\mathrm{Q}=Æ \mathrm{~V} / Æ \mathrm{t}$

Per effettuare il test di portata si aspirano $10 \mathrm{~mL}(\mathrm{~V})$ effettuando un conteggio del tempo impiegato $(t)$ in secondi (s) monitorando l'eventuale collabimento della vaschetta.

Si devono aspirare $10 \mathrm{~mL}$ di sangue nel più breve tempo possibile senza però assistere al collasso anche parziale della vaschetta.

Tale collasso indica una possibile occlusione o malfunzionamento del catetere e simula il successivo comportamento dello spezzone pompa dell'apparecchiatura per emodialisi in caso di difficoltà nell'aspirazione. Il test può essere ripetuto più volte.

La portata risultante, in assenza di collabimento della vaschetta, è riportata in Tabella I: (e.g. se si aspirano 10 $\mathrm{mL}$ in 3 secondi, la portata del lume è di $200 \mathrm{~mL} / \mathrm{min}$ ) il tempo impiegato al riempimento è inversamente proporzionale alla portata del lume:

$\begin{array}{ccc}\begin{array}{l}\text { Volume } \\ (\mathrm{ml})\end{array} & \begin{array}{l}\text { Time } \\ (\mathrm{s})\end{array} & \begin{array}{c}\text { Flowrate } \\ (\mathrm{ml} / \mathrm{min})\end{array} \\ 10 & 2 & 300 \\ 10 & 3 & 200 \\ 10 & 4 & 150 \\ 10 & 5 & 120 \\ 10 & 6 & 100 \\ 10 & 7 & 86 \\ 10 & 8 & 75 \\ 10 & 9 & 67 \\ 10 & 10 & 60\end{array}$

La nostra esperienza nell'uso del device Haemocatch

Dal $1^{\circ}$ agosto del 2009 al 31 gennaio 2010 abbiamo utilizzato il device Haemocatch su 38 CVC Bilume effettuando più di 2000 procedure di priming/attacco.
Il posizionamento del catetere era cosi suddiviso:

20 in giugulare interna

11 in succlavia

7 in femorale

Per una valutazione comparativa/funzionale del device è stata adottata per un periodo di un mese (sia con il device che in assenza), una semplice scheda (Tab. II) in cui si riportavano i parametri relativi alla portata dei lumi e alle difficoltà riscontrate nell'operatività stessa. La valutazione è stata effettuata sia utilizzando il device che con il metodo tradizionale.

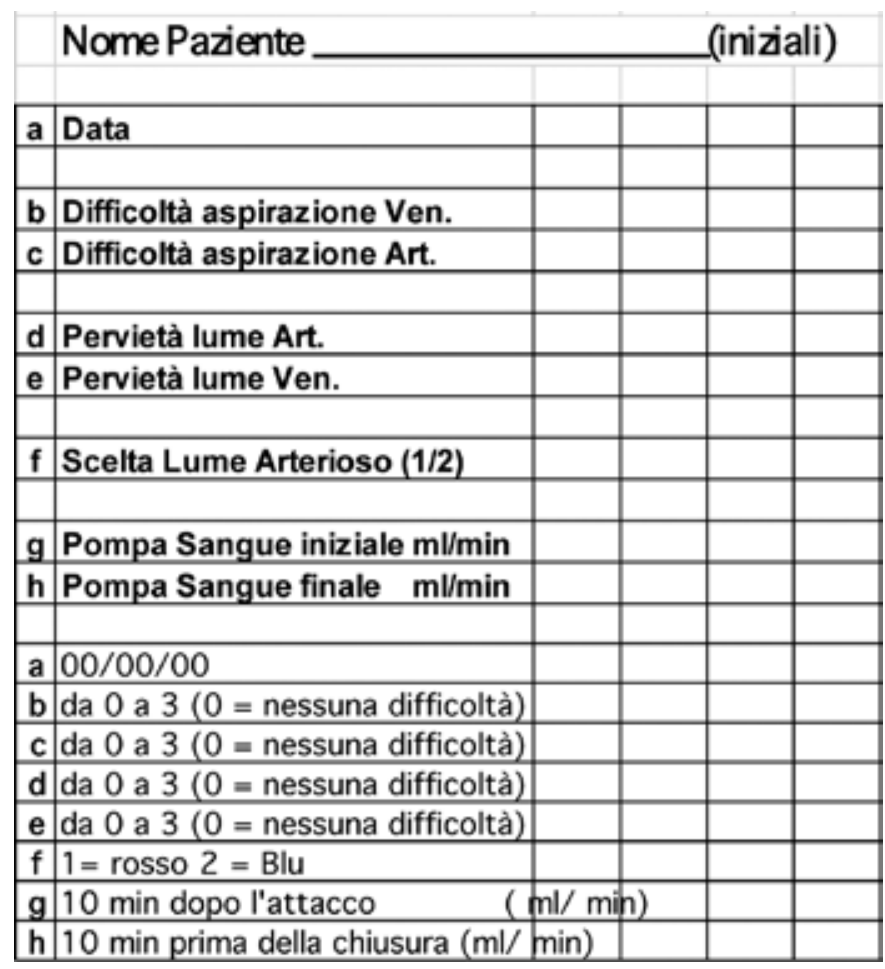

\begin{tabular}{|l|l|}
\hline 0 & Nessuna difficoltà/ottima pervietà \\
\hline 1 & Leggera difficoltà/buona pervietà \\
\hline 2 & Media difficoltà/pervietà scarsa \\
\hline 3 & Alta difficoltà/non pervio-occluso \\
\hline
\end{tabular}

Lo scopo della scheda era di verificare mediante un semplice conteggio, un eventuale miglioramento del trend della portata dell'accesso e degli eventuali eventi occlusivi o parzialmente occlusivi in seguito all'utilizzo del device in comparazione al metodo tradizionale da noi utilizzato. 
Da tale conteggio non è emersa una significativa differenza che possa portare a dire che l'utilizzo nel tempo (breve) del device Haemocatch abbia apportato un miglioramento nelle performance dell'accesso.

Bisogna però osservare che in questa valutazione numerica la soggettività, la manualità e l'esperienza dell'operatore giocano un ruolo molto importante.

E stato osservato però che nella maggior parte dei casi, il dispositivo è stato sconnesso quando l'attivazione manuale dello stantuffo della siringa ha evidenziato come il lume del catetere fosse pervio.

La pervietà del catetere è sempre stata confermata dal successivo utilizzo dello stesso.

In tutti i casi, oltre al lavaggio e riempimento del catetere è stato effettuato il "test di portata del catetere".

\section{Osservazioni}

Come richiesto dalla pratica clinica corrente, tutti i dispositivi sono stati ispezionati visivamente prima dell'apertura del confezionamento singolo. In tutti i casi, la busta non presentava segni di deterioramento o rotture e l'etichetta identificativa era chiaramente leggibile. I dispositivi utilizzati erano tutti perfettamente integri e utilizzabili.

Come tutti i dispositivi impiegati in campo ospedaliero, anche il dispositivo oggetto di questa relazione deve essere smaltito correttamente poiché al termine del suo utilizzo risulta contaminato.

Nella esperienza di questo centro, tutti i dispositivi hanno funzionato correttamente; le connessioni alla siringa per mezzo di standard luer si sono dimostrate efficaci in quanto a tenuta e collegamento pratico ed ergonomico tra il dispositivo e la siringa, non in dotazione.

Il dispositivo, nel corso del suo utilizzo, è sottoposto a stress meccanico dovuto alla variazione di pressione all'interno del dispositivo stesso, in conseguenza della movimentazione del pistone della siringa e della conseguente creazione di flusso. Quando la siringa viene svuotata, il dispositivo è sottoposto a pressione positiva, viceversa esso è sottoposto a pressione negativa quando lo stantuffo viene ritirato.

Il dispositivo può collabire all'altezza della vaschetta in caso di eccessiva pressione negativa.

E stato verificato nel nostro centro che in tutti i casi in cui il dispositivo era visivamente collabito, esso ha riacquistato la sua forma originaria non appena lo stress pressorio è stato eliminato.

L'utilizzo del dispositivo comporta l'estrazione dal circuito ematico di una certa quantità di sangue, corrispondente all'incirca al priming $(10 \mathrm{~mL})$ del dispositivo stesso.

Il volume di sangue contenuto nella siringa connessa al dispositivo può essere completamente restituito al paziente; al contrario, un certo volume di sangue rimane inevitabilmente all'interno del dispositivo al termine del suo utilizzo, nonostante sia generalmente possibile restituirne una certa quantità schiacciando manualmente il corpo della vaschetta.

Pertanto, l'effetto collaterale del dispositivo risulta essere la perdita di una certa quantità di sangue che abbiamo stimato essere poco meno di $10 \mathrm{~mL}$.

Tale effetto collaterale, comune anche alla pratica clinica corrente in cui il sangue viene versato in un contenitore aperto per visualizzare l'eventuale presenza di coaguli (circa $5 \mathrm{~mL}$ per lume), risulta essere accettabile dato il fatto che risulta essere comunque inferiore o al massimo sovrapponibile.

I riscontri che abbiamo effettuato rispetto al metodo tradizionale utilizzato e precedentemente illustrato sono interessanti.

Il dispositivo ha dimostrato, infatti, di svolgere efficacemente la funzione di priming, inteso come riempimento e "lavaggio", del catetere venoso centrale nei casi esaminati.

In particolare, è stato notato come le manovre contemplate nell'uso del device consentano di preparare il catetere per il successivo utilizzo (connessione alla apparecchiatura per emodialisi), riducendo potenzialmente il rischio di contaminazione dell'accesso venoso rispetto alla pratica clinica corrente.

Infatti, il lume del catetere può essere sottoposto ad alcuni passaggi di sangue senza che sia necessario disconnettere il dispositivo dal catetere stesso e questa operazione viene condotta in sicurezza poiché il sangue aspirato, mediante il circuito delle valvole passa per il filtro da 50 micron e viene re-infuso senza gli eventuali coaguli.

Un altro parametro interessante è la standardizzazione/ ripetitività del metodo, che consente che le valutazioni e il numero delle manovre siano guidate da una valutazione "più oggettiva" del funzionamento del catetere, in pratica che le stesse siano meno "operatore-dipendente".

Infatti premessa indispensabile di una buona seduta dialitica è un'adeguata portata dell'accesso vascolare; il device Haemocatch ci ha permesso di quantificare, prima dell'inizio della seduta dialitica, la portata del catetere per ogni singolo lume.

Pertanto, la seduta dialitica è stata sempre avviata solo dopo una constatazione oggettiva della portata del catetere riducendo quindi drasticamente l'evenienza dell'interruzione del trattamento per portata insufficiente. Infine il dispositivo consente di evitare di diffondere il sangue del paziente in campo aperto per visualizzare eventuali coaguli estratti dal lume del catetere, poiché il dispositi- 
vo è in grado di effettuare il filtraggio del sangue.

Quest'ultimo vantaggio diminuisce in modo considerevole le possibilità di contaminazione dell'operatore $\mathrm{e}$ dell'ambiente circostante ed esime l'operatore da una "visualizzazione" opinabile.

Per quanto riguarda il metodo e l'operatività, abbiamo avuto come riferimento per l'utilizzo le istruzioni per l'uso del dispositivo, dotate di illustrazioni, che sono sufficientemente chiare per un operatore professionale del campo dialitico.

Non sono stati verificati casi in cui il dispositivo, al termine dell'utilizzo, mostrasse segni di usura quali crepe, deformazioni o opacità.

Inoltre il dispositivo nel suo complesso ha dimensioni e forma tali da renderlo maneggevole e sicuro per l'utilizzo al letto del paziente.

In questa esperienza prettamente tecnica non è stata presa in considerazione una regolare valutazione sulla incidenza delle infezioni che, a una mera osservazione numerica, non ha sortito alcun elemento di significativo miglioramento o peggioramento.

\section{Conclusioni}

Da questa nostra, per quanto breve, esperienza, risulta che il dispositivo migliora lo stato dell'arte della manovra di preparazione e priming di un CVC.

La valutazione condotta nel nostro centro ha consentito di verificare come l'utilizzo del dispositivo aumenti la sicurezza sia del paziente che dell'operatore.

Il paziente viene sottoposto a un numero minore di manovre di connessione e sconnessione, diminuendo quindi la probabilità di una infezione dell'accesso venoso centrale.

Il rischio di contaminazione legato a questa manovra dovrebbe essere minimizzato da comportamenti corretti da parte del personale infermieristico, volti come in ogni manovra ospedaliera a minimizzare il rischio di infezione.

Inoltre l'operatore si trova ad operare con un dispositivo completamente chiuso con pareti trasparenti e pertanto il sangue estratto dal lume del catetere può essere ispezionato visivamente senza che esso rischi di venire accidentalmente a contatto con l'operatore e/o con altri pazienti. Rimane la necessità per l'operatore di connettere e sconnettere il dispositivo per una sola volta al catetere e questa operazione è di fatto ineliminabile e comune ad ogni manovra di lavaggio, preparazione e priming.

Si rende inoltre necessaria una valutazione effettuata sul lungo periodo per verificare se il device apporti vantaggi nelle performance del catetere in termini di portata.

Riteniamo pertanto che i vantaggi principali nell'utilizzo del device Haemocath nella configurazione da noi utilizzata, siano quelli legati alla standardizzazione del metodo di attacco al CVC e nella preliminare e oggettiva valutazione della portata dell'accesso.

E altresì auspicabile eseguire uno studio esteso atto a comparare e a verificare nel metodo di priming tradizionale e in quello attuale l'incidenza delle infezioni, che sono oggi l'elemento di discussione più rilevante nell'utilizzo dei CVC in emodialisi.

Tuttavia, secondo questa seppur breve esperienza, crediamo che l'utilizzo di un device dedicato che comporti un circuito chiuso, la riduzione/standardizzazione del numero connessioni e la valutazione della portata del CVC, possa apportare un miglioramento dell'"assessment" di una fase fondamentale quale è la preparazione e la valutazione funzionale dell'accesso vascolare prima della seduta dialitica.

\section{Riassunto}

Il catetere venoso centrale rappresenta uno dei possibili accessi vascolari per il paziente in emodialisi. I protocolli attualmente in uso per la procedura di attacco prevedono una serie di manovre di movimentazione del sangue seguite da ripetute connessioni-sconnessioni di alcune siringhe, con possibili rischi infettivi sia per il paziente sia per l'ambiente e con una valutazione soggettiva della portata dell'accesso. In questa breve esperienza abbiamo utilizzato un nuovo presidio, denominato "Haemocatch", che permette di aspirare il sangue da entrambi i lumi del catetere venoso centrale mediante una sola siringa, di restituire il sangue filtrato e privo di eventuali coaguli e di quantificare la portata dell'accesso prima dell'inizio della seduta dialitica in un sistema chiuso. I vantaggi di questo device innovativo sono la standardizzazione del metodo di priming, la riduzione del numero delle connessioni con conseguente minor esposizione al rischio di infezioni, la maggiore sicurezza sia per il paziente sia per l'operatore e la preliminare valutazione della portata dell'accesso, che fornisce un'importante informazione all'operatore e riduce l'evenienza di interruzione del trattamento per portata insufficiente.

Parole chiave: Catetere Venoso Centrale, CVC, Haemocatch, Priming, Test di Portata
Indirizzo degli Autori:
Donata Borin, MD
U.O.C. di Nefrologia e Dialisi
Ospedale di Legnago (VR)
donata.borin@aulsslegnago.it 


\section{Bibliografia}

1. Donovan K. Population requirements for vascular access surgery. Eur J Endovasc Surg 2006; 31 (2): 176-80.

2. Foundation NK. NKF-K/DOQI Clinical practice guidelines for vascular access: Update 2000. Am J Kidney Dis 2001; 37(Suppl 1): S137-81.

3. Pisoni RL, Young EW, Dykstra DM, et al. Vascular access use in Europe and United States Results from the DOPPS. Kidney Int 2002; 61: 305-16.

4. Pastan S, Soucie JM, McClellan WM. Vascular access and increased risk of death among hemodialysis patients. Kidney Int 2002; 62: 620-6.

5. Charmaine E. Lok, Debra Appleton, Cynthia Bhola, Brian Khoo, Robert M. A. Richardson. Trisodium citrate $4 \%$ - an alternative to heparin capping of haemodialysis catheters. Nephrol Dial Transplant 2007; 22: 477-83.

6. Grudzinski L, Quinan P, Kwok S, Pierratos A. Sodium citrate $4 \%$ locking solution for central venous dialysis catheters - an effective, more cost-efficient alternative to heparin. Nephrol Dial Transplant 2007; 22: 471-6.
7. Michaud D, Komant T, Pfefferle P. Four percent trisodiumcitrate as an alternative anticoagulant for maintaining patency of central venous hemodialysis catheters: case report and discussion. Am J Crit Care 2001; 10: 351-4.

8. Hendrickx L, Kuypers D, Evenepoel P, Maes B, Messiaen T, Vanrenterghem Y. A comparative prospective study on the use of low concentrate citrate lock versus heparin lock in permanent dialysis catheters. Int J Artif Organs 2001; 24: 208-11.

9. Warkentin TE, Levine MN, Hirsh J, et al. Heparin-induced thrombocytopenia in patients treated with low-molecularweight heparin or Unfractionated Heparin. N Engl J Med 1995; 332: 1330-6.

10. Karaaslan H, Peyronnet P, Benevent D, Lagarde C, Rince M, Leroux-Robert C. Risk of heparin lock-related bleeding when using indwelling venous catheter in haemodialysis. Nephrol Dial Transplant 2001; 16: 2072-4.

11. Polaschegg HD, Sodemann K. Risks related to catheter locking solutions containing concentrated citrate. Nephrol Dial Transplant 2003; 18: 2688-90. 